\title{
Canonical BAF Complex
}

National Cancer Institute

\section{Source}

National Cancer Institute. Canonical BAF Complex. NCI Thesaurus. Code C156919.

A SWI/SNF family chromatin-remodeling complex comprised of at least ACTB, ARID1A/BAF250A or ARID1B/BAF250B, SMARCA2/BRM and/or SMARCA4/BRG1/BAF190A, ACT L6A/BAF53 or ACT L6B/BAF53B, DPF1/BAF45B, DPF2/BAF45D or DPF3/BAF45C, SMARCE1/BAF57, SMARCC1/BAF155, SMARCC2/BAF170, SMARCB1/SNF5/INI1, BCL7A, BCL7B or BCL7C, and SMARCD1/BAF60A, SMARCD2/BAF60B or SMARCD3/BAF60C. 